\title{
Weniger ist manchmal mehr
}

\begin{abstract}
_ Ältere Menschen nehmen oft viele verschiedene Medikamente ein. Im Rahmen einer individualisierten Therapie sollte jedoch regelmäßig überprüft werden, ob die Medikation tatsächlich angemessen ist. Vor rund zwei Jahren wurde das Projekt InTherAKT zur Verbesserung der medikamentösen Versorgung in Altenhilfeeinrichtungen gestartet [www.interakt.de]. Herzstück der gemeinsamen Arbeit von 15 Hausärzten,
\end{abstract}

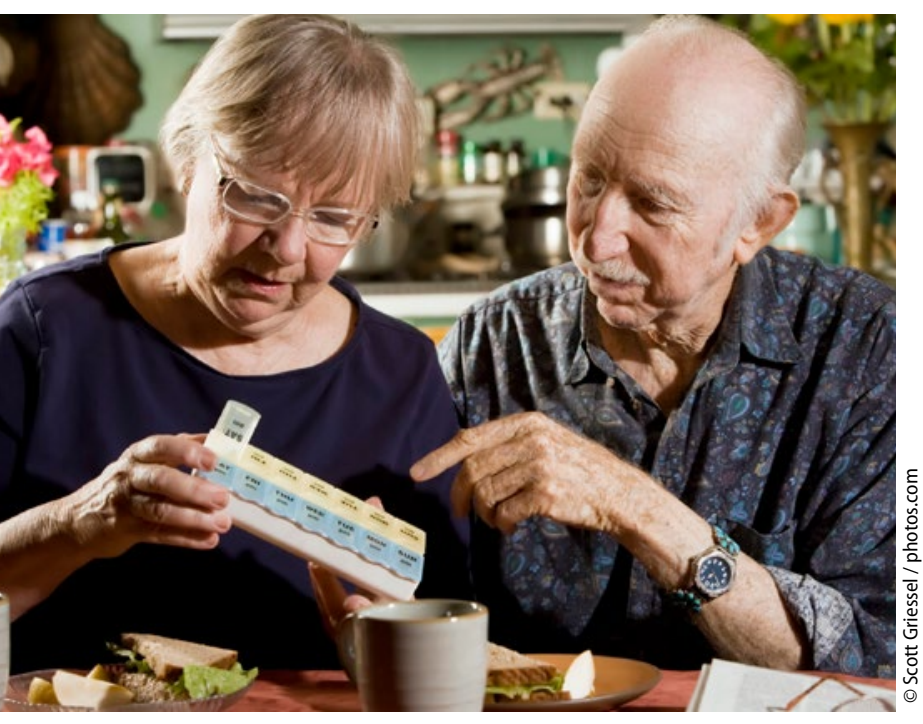

Müssen alle diese Tabletten wirklich sein? zwölf Apothekern und dem Pflegepersonal an zehn Altenhilfeeinrichtungen im Raum Münster ist eine Online-Plattform, die inzwischen zum Patent angemeldet wurde. Seit Anfang 2016 wurde es an rund 90 Patienten überprüft und optimiert.

Wie der Projektleiter Prof. Jürgen Osterbrink, Salzburg, erläuterte, seien betagte und hochbetagte Menschen besonders häufig von unerwünschten Arzneimittelinteraktionen betroffen. Vielfach seien die Interaktionen das Resultat nicht abgestimmter Prozesse der Leistungserbringer. Ein Ziel des Projektes InTherAKT besteht deshalb darin, die Kommunikation und Kooperation zwischen den Berufsgruppen zu optimieren. Dazu wurden alle Beteiligten nicht nur online, sondern auch in Präsenzveranstaltungen geschult.

$\mathrm{Zu}$ den gravierendsten Folgen unerwünschter Arzneimittelwirkungen im Alter zählen aus Sicht von Prof. Georg Hempel,
Münster, Gangunsicherheit, Synkopen und Stürze, die nicht selten lange Krankenhausaufenthalte und den Verlust der Autonomie nach sich ziehen. Eine ständige kritische Überprüfung der Medikation sei deshalb gerade bei älteren Menschen von großer Bedeutung.

\section{Bis zu fünf Medikamente gestrichen} In InTherAKT wird die Angemessenheit der Medikation anhand des „Medication Appropriateness Index" (MAI) erhoben - und hier ergab sich eine Verbesserung um durchschnittlich $25 \%$. Besonders deutlich zeigte sich der Erfolg bei Patienten, die vorher sehr viele Medikamente einnehmen mussten: „In solchen Fällen konnten wir eine Verbesserung um bis zu $60 \%$ beobachten", berichtete der Pharmazeut. Im Einzelfall konnte die Zahl der Medikamente um bis zu fünf Präparate reduziert werden. Damit unterstreichen die Ergebnisse die Notwendigkeit der interprofessionellen Zusammenarbeit, bei der jede Berufsgruppe ihre Kompetenzen einbringt.

\section{Dr. Matthias Herrmann}

- Lunch-Symposium „Polypharmazie in der Altenhilfe - Learnings auch für die Schmerztherapie", Deutscher Schmerzkongress; Mannheim, Oktober 2017 (Veranstalter: Grünenthal)

\section{Erektile Dysfunktion}

\section{Tadalafil-Generikum verfügbar}

_ Hormosan Pharma erweitert sein Produktportfolio: Mit Tadalafil-Hormosan steht ab 15. November 2017 in Deutschland ein weiterer PDE-5-Hemmer zur Behandlung der erektilen Dysfunktion zur Verfügung.

Bisher ist Hormosan im Bereich erektile Dysfunktion mit SildenafilHormosan als einer der preisgünstigsten und absatzstärksten Anbieter von Sildenafil bekannt. Mit der Neueinfüh- rung von Tadalafil-Hormosan bedient der Generika-Spezialist die individuellen Bedürfnisse der Betroffenen mit erektiler Dysfunktion. Männer, die eine Wirkdauer von bis zu 36 Stunden vorziehen, präferieren mitunter Tadalafil gegenüber anderen PDE-5-Hemmern. Auch der schnelle Wirkeintritt von 15-30 Minuten sowie die uneingeschränkte Wirkung von Tadalafil auch nach reichhaltigem Essen ist für Viele bisweilen ein ausschlaggebendes Kriterium bei der Wahl der Präparate.

Das Generikum zeichnet sich durch eine deutliche Preisersparnis gegenüber dem Originalpräparat, Tabletten in originalnaher Farbe sowie einer zusätzlichen Packungsgröße von 24 Tabletten bei der meistverkauften 20-mg-Stärke aus.
Red. 